Int. J. Odontostomat., 8(3):469-474, 2014.

\title{
Rehabilitación de los Dientes Anteriores con el Sistema Cerámico Disilicato de Litio
}

\author{
Rehabilitation of Anterior Teeth with Ceramic Lithium Disilicate System
}

\begin{abstract}
Rolando Ignácio Figueroa*;Fernando Goulart Cruz*; Rodrigo Furtado de Carvalho*; Fabíola Pessoa Pereira Leite ${ }^{* \star \star} \&$ Maria das Graças Afonso de Miranda Chaves ${ }^{\star * \star *}$
\end{abstract}

FIGUEROA, R I.; CRUZ, F. G.; DE CARVALHO, R. F.; LEITE, F. P. P. \& CHAVES, M. G. A. M. Rehabilitación de los dientes anteriores con el sistema cerámico disilicato de lítio. Int. J. Odontostomat., 8(3):469-474, 2014.

RESUMEN: Las cerámicas dentales son conocidas por su excelencia en reproducir artificialmente los dientes naturales. Casos clínicos más severos en el sector anterior, con acentuada alteración del color, necesidad de substitución de coronas o amplias restauraciones, asociado a una redefinición y largura de los dientes, son solucionadas satisfactoriamente a través de los nuevos sistemas cerámicos como lo sistema IPS e.Max. Este sistema es basado en una cerámica vítrea reforzada con cristales de disilicato de litio (60-65\%). En este artículo se reporta un caso de una paciente de 47 años de edad que se realizaron cuatro coronas totales con el sistema cerámico IPS e.Max. El resultado final muestra una estética natural y funcional, con óptimas propiedades ópticas, que son peculiares al sistema cerámico empleado. La conclusión es que el sistema cerámico IPS e.Max es un sistema muy versátil, además de convertirse actualmente en una excelente alternativa como sistema de restauración, tanto estética como funcionalmente, consiguiendo una excelente armonía entre la sonrisa del paciente y la estética de las restauraciones.

PALABRAS CLAVE: cerámicas dentales, estética, disilicato de lítio, coronas, dientes anteriores.

\section{INTRODUCCIÓN}

Un elevado porcentaje de los tratamientos que realiza el profesional en la consulta odontológica responde a la necesidad de reponer dientes anteriores. En aquellos casos en los que el empleo de la prótesis fija esté indicado, el profesional debe conocer los materiales restauradores que se encuentran a su disposición.

Las cerámicas dentales son conocidas por su excelencia en reproducir artificialmente los dientes naturales. En el siglo XVIII fue empleada por primera vez en la odontología como diente artificial para prótesis totales. A partir del siglo XX pasó a ser utilizada para la confección de restauraciones metal - cerámicas y más recientemente con el desarrollo de la tecnología cerámica surgieron las restauraciones libres de metal (Gomes et al., 2008). Han sido tan importantes y revo- lucionarios los cambios y aportes en este campo en los últimos 20 años que en la actualidad existe una multitud de sistemas cerámicos (Martínez Rus et al., 2007).

La estética es un concepto subjetivo, sometido a grandes cambios según el medio socio-cultural que se trate. Pero no cabe duda de que en el entorno en que nos movemos, hablar de restauraciones estéticas en el momento actual, implica hablar de cerámica sin metal. Además, las porcelanas son más inertes que los metales. Sabemos que las aleaciones pueden verter iones nocivos al medio oral al sufrir corrosión, hecho que no ocurre en las cerámicas debido a su baja reactividad química (Martínez Rus et al.). Casos clínicos más severos en la sector anterior, con acentuada alteración del color, necesidad de substitución de co-

Universidad de La Frontera, Temuco, Chile.

** Magíster en Clínica Odontológica, Universidad Federal de Juiz de Fora, Minas Gerais, Brasil.

*** Doctor en Prótesis Dentaria, Universidad Estadual Paulista Júlio de Mesquita Filho; Profesor en la Universidad Federal de Juiz de Fora, Minas Gerais, Brasil.

Doctor en Biopatologia Bucal en la Universidad Estadual Paulista Júlio de Mesquita Filho; Profesor en la Universidad Federal de Juiz de Fora, Minas Gerais, Brasil. 
ronas o amplias restauraciones, asociado a una redefinición y largura de los dientes, son solucionadas satisfactoriamente a través de los nuevos sistemas cerámicos (Ivoclar Vivadent, 2014).

La creciente demanda de pacientes por restauraciones estéticamente agradables ha impulsado la búsqueda de diferentes materiales con propiedades ópticas mejoradas que podrían ser utilizados para tales tipos de restauraciones (Ereifej, 2009).

Hace más de 15 años nació un sistema cerámico Ilamado IPS Empress I (Ivoclar North América, Amherst, NY, EUA), el que es basado en una cerámica vítrea reforzada con cristales de leucita (35-55\% Sistema IPS Empress I - E1) para posteriormente ser reforzado por cristales de disilicato de litio $(60-65 \%$ Sistema IPS Empress II - E2) (Gomes et al.; Heintze et al., 2008), en la cual la cerámica es inyectada en un molde de revestimiento obtenido por la técnica de la cera perdida, bajo alta temperatura y presión. El sistema IPS Empress simplificó el problema de la contracción durante la quema de la cerámica, común para las cerámicas feldespáticas, debido a la alta presión de inyección de la cerámica en el molde a altas temperaturas (Heintze et al.; Lagos Flores et al., 2007).

En el año 2007 surge el sistema IPS E-max press /CAD el cual esta reforzado solo con cristales de disilicato de litio pero mejorando la transparencia y translucidez y así aumentando la estética, no obstante, ofrecen una resistencia a la fractura mayor que Empress $®$ II debido a una mayor homogeneidad de la fase cristalina. Al igual que en el sistema anterior, sobre estas cerámicas se aplica una porcelana feldespática convencional para realizar el recubrimiento estético mediante la técnica de capas (Martínez Rus et al.; Heintze et al.).

Cada paciente comprende sus propias exigencias y sus propios objetivos. Debido a los dos componentes del sistema satisface prácticamente todas las exigencias (Clavijo et al., 2007; Ivoclar Vivadent):

- Los componentes para la tecnología de Inyección incluyen las pastillas altamente estéticas de cerámica vítrea de disilicato de litio IPS e.max Press y las pastillas de cerámica vítrea de fluorapatita IPS e.max ZirPress para una eficiente técnica de inyección sobre óxido de circonio.

- De acuerdo a las exigencias de cada caso están disponibles dos tipo de material para la técnica CAD/CAM, los innovadores bloques de cerámica vítrea de disilicato de litio IPS e.max CAD y el altamente resistente óxido de circonio IPS e.max ZirCAD.

La cerámica de estratificación de nanofluoarapatita IPS e.max Ceram, que es utilizada como material de estratificación y de caracterización para todos los componentes de IPS e.max, sean de base vítrea o circonio.

Estas posibilidades del uso de IPS e.max lo convierte en un sistema totalmente flexible para los rehabilitadores, además de permitir que los cuatro materiales de estructuras diferentes puedan ser estratificados con la ya mencionada IPS e.max Ceram, la cual garantiza un biomimetismo con la estructura dentaria (Clavijo et al.; Ivoclar Vivadent).

Por lo anteriormente expuesto, este artículo relata el devolución de la sonrisa con el sistema IPS e.Max Press, presentando el tratamiento protésico mediante la confección de 4 coronas cerámicas en el sector antero superior

\section{CASO CLÍNICO}

Paciente de sexo femenino, 47 años de edad, asiste a la clínica de prótesis fija de la Facultad de Odontología de la Universidad Federal de Juiz de Fora, que como motivo de consulta era la queja por la apariencia estética de su sonrisa.

Al examen clínico se observó la presencia de extensas restauraciones de resina compuesta fotopolimerizable filtradas y pigmentación oscuras en los dientes 11, 12, 21 y 22 (Fig. 1). Radiográficamente se evidenció que los cuatro incisivos superiores estaban despulpados, cuyos tratamientos endodónticos se observaban en buen estado. Por lo anteriormente expuesto se optó por la colocación y cementación de espigas de fibra de vidrio (WhitePost DC, FGM, Brasil) en los dientes 11 y 12 para la continuidad del tratamiento restaurador (Fig. 2). Con la finalidad de obtener un excelente estado tanto estético como funcional se escogió la confección de cuatro coronas totales unitarias con el sistema IPS e.Max Empress (Ivoclar Vivadent, Alemania)

Después de realizar completamente la planificación e interacción entre el clínico, paciente y laboratorista, se inició el desgaste selectivo de la es- 


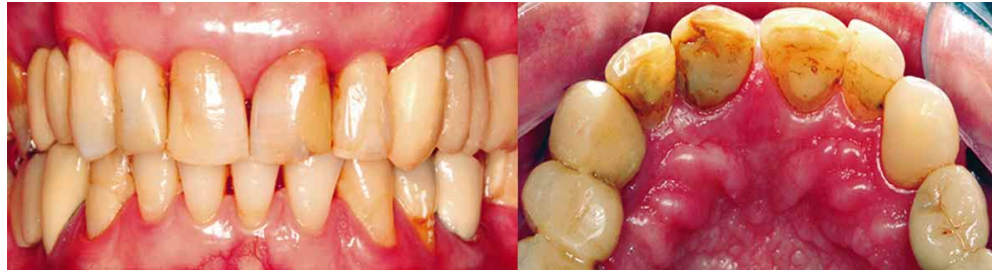

Fig. 1. Aspecto clinico iniciale en los dientes 11, 12, 21 y 22.

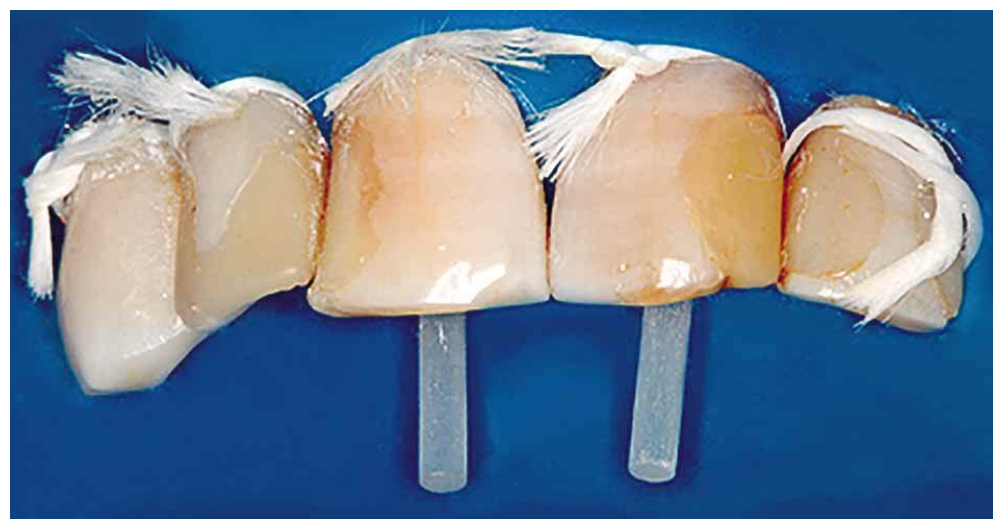

Fig. 2. Colocación y cementación de espigas de fibra de vidrio (WhitePost DC, FGM, Brasil) en los dientes 11 y 12 para la continuidad del tratamiento restaurador.

tructura dental. Fueron realizados surcos de orientación con la punta diamantada 3098MF (KG Sorensen), respetando la inclinación de los tercios cervical, medio e incisal de los dientes.

A continuación se unieron los surcos de orientación de los preparos con la misma fresa, realizando una terminación marginal en hombro redondeado. El acabamiento del margen gingival fue realizado con instrumentos manuales MA-2 (SafidentCosmedent-Brasil(®), lo que permite un óptimo refinamiento de la región cervical, finalizando de esta forma la preparación de los muñones (Fig. 3).

Para las impresiones, se decidió utilizar silicona de adición Optosil/Xantopren (Kulser, Alemanha) (Fig. 4), para la separa-

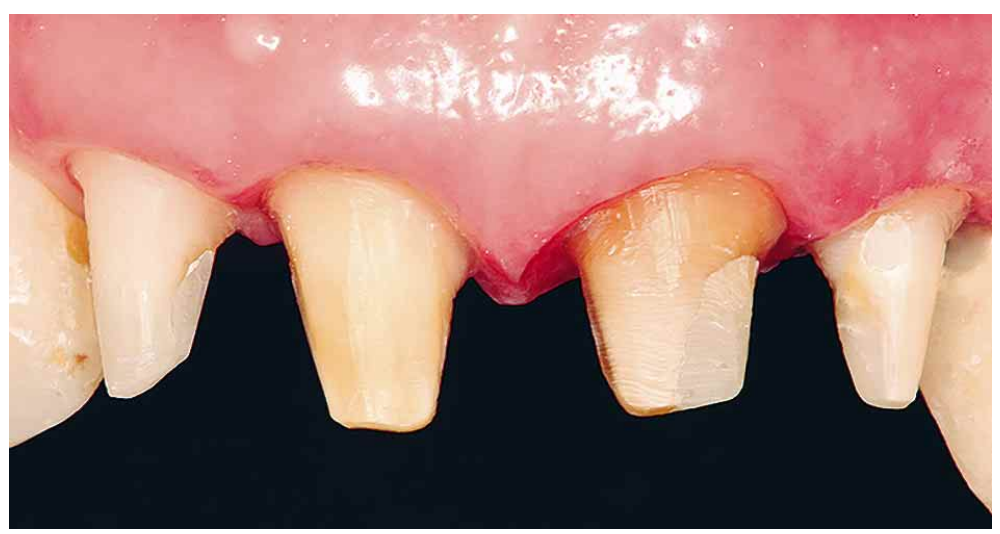

Fig. 3. Forma la preparación de los muñones dentarios. ción gingival se utilizó la técnica de doble hilo, realizada con hilo 000 y 0 Ultrapack (Ultradent-Oraltech-Brasil) (Fig. 4). El hilo 000 se mantuvo dentro del surco durante la toma de impresión, y fue removido solo el hilo 0 . A continuación se procedió a la selección del color, seguido de la cementación de los provisorios confeccionados con un compuesto de Bisacrilico (Luxatemp, DMG, Estados Unidos) (Fig. 4). Los casquetes fueron confeccionados con el sistema IPS e.Max Press (Fig. 5).

En la siguiente sesión fue realizada la cementación de las restauraciones. Luego de realizar aislamiento absoluto modificado y profilaxis con piedra pómez y cepiIlo ICB (Ultradent-Oraltech-Brasil), se procedió a la colocación de hilo retractor 000 y verificación de la adaptación de las coronas protésicas y la preparación previa a la cementación. Las restauraciones recibieron como tratamiento de superficie: condicionamiento interno con ácido fluorhídrico al $10 \%$ por 60 segundos (Acondicionador de porcelana - Dentsply, Brasil), lavado, y secado con chorros de aire/agua y la aplicación del agente de unión silano (CLEARFIL CERAMIC PRIMER - Kuraray Medical Inc., Okayama, Japón) que se ha secado por completo después de la aplicación, para seguir adelante con la cementación según la indicación del fabricante.

Para el acondicionamiento de las estructuras dentales se utilizó la mezcla de líquidos ED PRIMER A y ED PRIMER B, lo que es aplicado con un pincel microbrush a la superficie del diente en forma activa durante 60 segundos.

A continuación fue manipulado el cemento resinoso y la corona fue cementada a la preparación dentaria, pasados 120 segundos se realizó inmediatamente la remoción de los excesos. Cada cara de la corona fue fotocurada durante 40 segundos por una unidad de curado Optilux 501 (Demetón, Kerr) la intensidad de emisión de luz fue de $850 \mathrm{~mW} / \mathrm{cm}^{2}$.

El ajuste final de la oclusión fue realizado luego de la remoción del aislamiento 


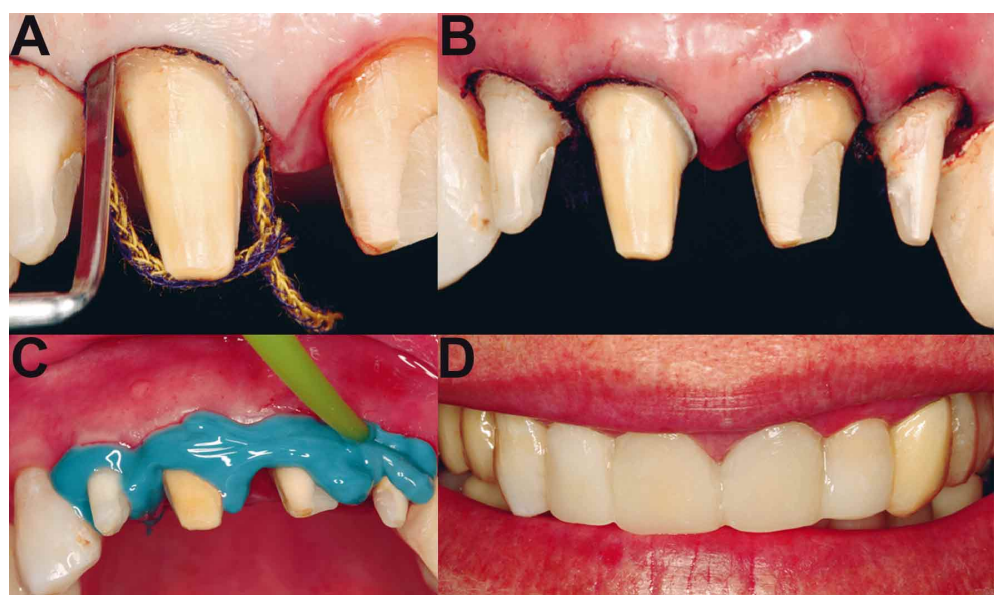

Fig. 4. Para la separación gingival se utilizó la técnica de doble hilo, realizada con hilo 000 y 0 Ultrapack (Ultradent-Oraltech-Brasil); C. Para las impresiones, se decidió utilizar silicona de adición Optosil/Xantopren (Kulser, Alemanha). D. Los provisorios confeccionados con un compuesto de Bisacrilico (Luxatemp, DMG, Estados Unidos).

del campo operatorio, donde también se hizo el pulido post-glace de la restauración, para la remoción del brillo en exceso de las restauraciones, optimizando aun más la naturalidad de las coronas.

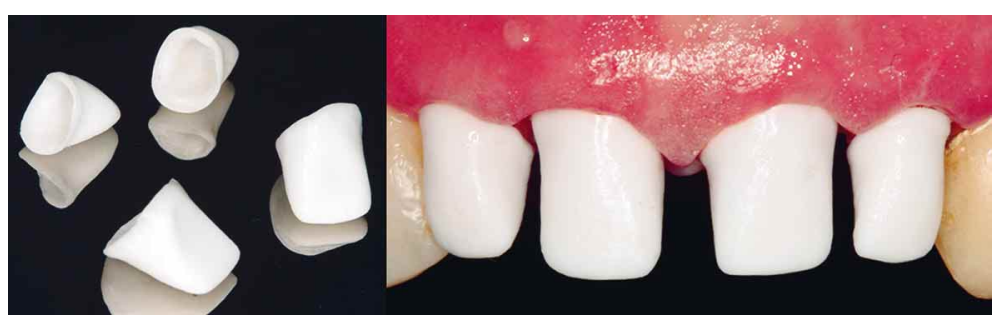

Fig. 5. Los casquetes de las coronas.

El resultado final muestra una estética natural y funcional, con óptimas propiedades ópticas, que son peculiares al sistema cerámico empleado, además de la completa integración de las restauraciones con la sonrisa del paciente (Fig. 6).

\section{DISCUSIÓN}

La Real Academia Española define algo estético cuando tiene armonía y presenta una apariencia agradable a la vista, por eso en un tratamiento rehabilitador se debe devolver una sonrisa armoniosa, que sea de acuerdo a las características estéticas propias de cada paciente, en los tiempos que se viven hoy en día no solo basta con la devolución de la forma y la función de los dientes.

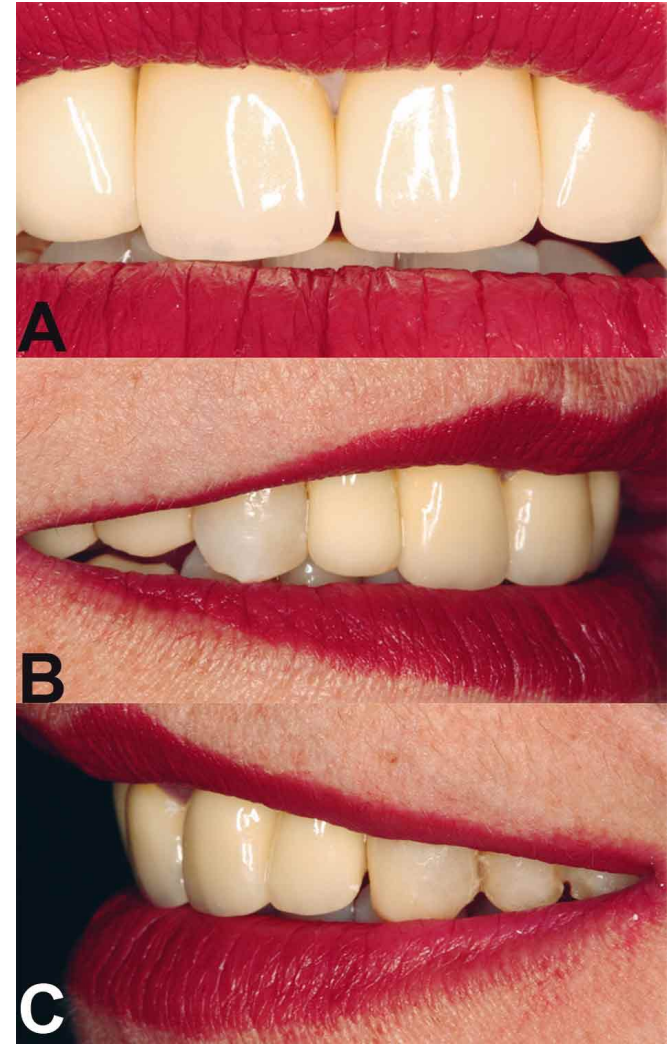

Fig. 6. Aspecto clínico final.

El caso visto anteriormente fue rehabilitado con el sistema cerámico IPS e.Max Press, el cual presenta una técnica de inyección de la cerámica. Para la técnica de inyección existen dos tipos de pastillas cerámicas: IPS e.Max Zirpress, una cerámica a base de óxido de circonio; además del sistema IPS e.Max Press una cerámica de disilicato de litio. A ambos sistemas posteriormente se les aplica una cerámica de cobertura con base de vidrio, propiciando la estética deseada (Clavijo et al.; Martínez Rus et al.).

El circonio es más resiste que el disilicato de litio, por lo que en el sector anterior no se necesita utilizar tal resistencia, pero el disilicato de litio resiste hasta $400 \mathrm{mPa}$ (2008 MIRJANA) y la fuerza incisal máxima relatada en la literatura varia de 90 $\mathrm{N}$ a $370 \mathrm{~N}$ (Yildirim et al., 2003).

Otro motivo por el que se escogió este sistema es la estética que posee el 
disilicato de litio en comparación con el zirconio, ya que la translucidez es mucho mayor, lo que la convierte en un material mucho más estético con una apariencia más armónica y natural, así el circonio es opaco y se requiere una mayor cantidad de porcelana de cobertura para conseguir encubrir el casquete (Gehrke et al., 2010). Este material puede ser utilizado para realizar inlays, onlays, chapas, puentes de tres unidades. Las ventajas de este procedimiento es que se hace una mínima invasión, salud gingival, procedimiento fácil y de bajo costo. Tiene buena rigidez y una buena calidad óptica (Boskovic et al., 2008).

Se optó por la utilización del sistema IPS e.Max Press por sobre el sistema CAD debido a que se ha demostrado una mejor longevidad en comparación a este sistema, esto se basa en los estudios de Guess et al. (2006), donde la restauración hecha con CAD/ CAM se observó una tasa de supervivencia de un $97 \%$ y de un $100 \%$ para el sistema IPS e-Max Press en un tiempo de 24 meses.

Una de las ventajas del sistema IPS e.Max es que presenta una cerámica de cobertura (IPS e.Max Ceram) la cual es con base de fluorapatita y sirve para estratificar todos los tipos de cerámicas, independiente de ser de disilicato de litio o de óxido de circonio, inyectable o CAD/CAM. Además de la variabilidad del sistema, presenta una excelente estética, garantizando las restauraciones cerámicas con buenas propiedades ópticas tales como translucidez y fluorescencia, muy similar a las de la propia estructura dentaria (Clavijo et al.; Martínez Rus et al.; Gomes et al.).

La buena estabilidad de este sistema cerámico frente a las adversidades del medio intra oral fue otro factor determinante en la elección del sistema cerámico para la rehabilitación del sector anterior para que el caso tuviese una buena durabilidad. Esto fue comprobado por un estudio de Samra et al. (2008) donde comparó la estabilidad del color de diferentes sistemas restauradores tanto directos como indirectos, sumergidos en una solución de café, y constató el mejor comportamiento del sistema IPS e.Max por sobre los otros.

El proceso de cementación es clave para la longevidad y el éxito clínico consiguiente. Cementos de resina tienen menor solubilidad en el medio oral, una mayor resistencia a la flexión, mejor estética, y una mejor eficacia en relación a los diferentes sustratos en comparación con otros agentes cementante (Bottino et al., 2009).
Un estudio realizado por Heintze et al. encontró que la resistencia a la fractura de las coronas e-Max Press que fueron cementadas con adhesivo fue del $42 \%$ superior a la media de fracturas por carga en comparación a coronas e.max Press cementadas con ionomero de vidrio. La prueba de este hecho es confirmado por estudios en laboratorio sobre fracasos clínicos de este material, teniendo en cuenta las características de la fractura en coronas e.Max, durante la carga dinámica donde invariablemente se produjeron las fracturas fue en la interfaz cemento - cerámica, lo que llevó a fracturas completas de la restauración con un patrón de media luna (Kelly et al., 1990; Thompson et al., 1994).

\section{CONCLUSIONES}

El sistema cerámico IPS e.Max es un sistema muy versátil, además de convertirse actualmente en una excelente alternativa como sistema de restauración, tanto estética como funcionalmente, consiguiendo una excelente armonía entre la sonrisa del paciente y la estética de las restauraciones.

Cabe destacar la importancia del manejo de la técnica en todos sus pasos además del trabajo en conjunto que se realiza con el laboratorista para conseguir un éxito seguro en la rehabilitación del paciente.

FIGUEROA, R I.; CRUZ, F. G.; DE CARVALHO, R. F.; LEITE, F. P. P. \& CHAVES, M. G. A. M. Rehabilitation of anterior teeth with ceramic lithium disilicate system. Int. J. Odontostomat., 8(3):469-474, 2014.

ABSTRACT: Dental ceramics are known for their excellence in the artificial reproduction of natural teeth. Clinical cases with severe marked discoloration in the anterior region, need of crown replacement or extensive restorations associated to a redefinition and length of the teeth, are successfully treated with new ceramic systems such as IPS system e. Max. The system is based on glassreinforced ceramic lithium disilicate crystals (60-65\%). This article describes a case of a 47 year old woman with four full crowns made with IPS e.max ceramic system is reported. The final result shows a natural and functional aesthetic with excellent optical properties, which are particular to the ceramic system used. In conclusion the IPS e.max ceramic system is a versatile system in addition to currently becoming an excellent alternative for restoration systems, both aesthetically and functionally, achieving an excellent balance between the patient's smile and aesthetic restorations.

KEY WORDS: dental ceramics, aesthetics, lithium disilicate, crown, anterior teeth. 


\section{REFERENCIAS BIBLIOGRÁFICAS}

Boskovic, M.; Stankovic, S.; Ajdukovic, Z. \& Krunic, N. Short review of non-metal ceramic systems. Acta Stomatol. Naissi, 24(57):767-73, 2008.

Bottino, M. A.; Faria, R. \& Valandro, L. F. Percepção - Estética em Próteses Livres de Metal em Dentes Naturais e Implantes. São Paulo, Artes Médicas, 2009. pp.469-542.

Clavijo, V. G. R.; de Souza, N. C. \& de Andrade, M. F. IPS e.Max: harmonização do sorriso. Rev. Dental Press Estét. (Maringá), 4(1):33-49, 2007

Ereifej, N.; Silikas, N. \& Watts, D. C. Edge strength of indirect restorative materials. J. Dent., 37(10):799-806, 2009.

Gehrke, S. A.; dos Santos, P. C. V.; Carvalho, N. T. A.; de Mello, R. M. \& Carbonari, M. J. metalfree sobre implante: parafusada ou cimentada - demonstração laboratorial e clínica. Full Dent. Sci., 1(3):248-53, 2010.

Gomes, E. A.; Assuncao, W. G.; Rocha, E. P. \& Santos, P. H. Cerâmicas Odontológicas: o estado atual. Ceram., 54(331):319-25, 2008.

Guess, P. C.; Stappert, C. F. \& Strub, J. R. Preliminary clinical results of a prospective study of IPS e.max Press- and Cerec ProCAD- partial coverage crowns. Schweiz. Monatsschr. Zahnmed., 116(5):493-500, 2006.

Heintze, S. D.; Cavalleri, A.; Zellweger, G.; Büchler, A. \& Zappini, G. Fracture frequency of all-ceramic crowns during dynamic loading in a chewing simulator using different loading and luting protocols. Dent. Mater., 24(10):1352-61, 2008.

Ivoclar Vivadent. IPS e.MAX Press. 2014. Disponible en: http://www.ivoclarvivadent.es

Lagos Flores, E.; Solá Ruiz, M. F.; Granell Ruiz, M. \& Román Rodríguez, J. L. Estudio clínico longitudinal de los puentes de IPS-Empress 2. Resultados a los 6 años. Rev. Int. Prótes. Estomatol., 9(3):199-206, 2007.

Martínez Rus, F.; Pradíes Ramiro, G.; Suárez García, M. J. \& Rivera Gómez, B. Cerámicas dentales: clasificación y criterios de selección. RCOE, 12(4):253-63, 2007.

Samra, A. P.; Pereira, S. K.; Delgado, L. C. \& Borges, C. P. Color stability evaluation of aesthetic restorative materials. Braz. Oral Res., 22(3):205-10, 2008.

Yildirim, M.; Fischer, H.; Marx, R. \& Edelhoff, D. In vivo fracture resistance of implant-supported all-ceramic restorations. J. Prosthet. Dent., 90(4):325-31, 2003.
Dirección para Correspondencia:

Maria das Graças Afonso de Miranda Chaves

Rua Halfeld, n 828 - 311.

Centro, Juiz de Fora

Minas Gerais, CEP: 36010-003

BRASIL

Email: duque05@gmail.com

Recibido: 04-02-2014

Aceptado: 23-10-2014 